Proceedings of the 8th Workshop on Quantum Chaos and Localisation Phenomena, May 19-21, 2017, Warsaw, Poland

\title{
Analysis of Missing Level Statistics for Microwave Networks Simulating Quantum Chaotic Graphs Without Time Reversal Symmetry - the Case of Randomly Lost Resonances
}

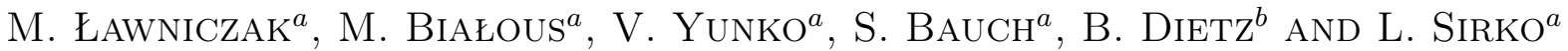 \\ ${ }^{a}$ Institute of Physics, Polish Academy of Sciences, Aleja Lotników 32/46, 02-668 Warszawa, Poland \\ ${ }^{b}$ School of Physical Science and Technology, and Key Laboratory for Magnetism and Magnetic Materials of MOE, \\ Lanzhou University, Lanzhou, Gansu 730000, China
}

\begin{abstract}
We present experimental and numerical studies for level statistics in incomplete spectra obtained with microwave networks simulating quantum chaotic graphs with broken time reversal symmetry. We demonstrate that, if resonance frequencies are randomly removed from the spectra, the experimental results for the nearest-neighbor spacing distribution, the spectral rigidity and the average power spectrum are in good agreement with theoretical predictions for incomplete sequences of levels of systems with broken time reversal symmetry.
\end{abstract}

DOI: 10.12693/APhysPolA.132.1672

PACS/topics: 05.40.--a, 05.45.Mt, 05.45.Tp, 03.65.Sq

\section{Introduction}

New achievements in the theory of quantum chaotic systems [1-3] have accounted for better understanding of experimental results obtained in real physical systems in the presence of energy losses (absorption, openness) and missing energy levels. It is established that the fluctuations in the spectra of quantum chaotic systems coincide with those of the eigenvalues of random matrices [4] from the Gaussian orthogonal ensemble (GOE) and the Gaussian unitary ensemble (GUE) for classically chaotic systems with and without time-reversal symmetry (TRS), in accordance with the Bohigas-Giannoni-Schmit (BGS) conjecture [5]. The energy levels of quantum systems with classically regular dynamics behave as if they were drawn from a Poissonian random process [6].

A multitude of theoretical and numerical studies devoted to the problems of quantum and wave chaos have been performed to date, yet not all non-generic features in the spectra of the real physical systems are fully understood. Experimental approaches to an understanding of these unsolved problems are feasible with microwave cavities and networks simulating two-dimensional (2D) quantum billiards [7-14] and one-dimensional (1D) quantum graphs [15-20], respectively. The formal analogies between the scalar Helmholtz equation, the Telegraph equation and the two- and one-dimensional Schrödinger equations, respectively, allow for it. Wave chaos was also studied using three-dimensional (3D) microwave cavities [21] but there is no direct analogy between the 3D vectorial Helmholtz equation and the Schrödinger equation. One should point out that the introduction of one-dimensional microwave networks simulating quantum graphs promoted considerably the experimental understanding of the features of quantum chaotic systems. Other systems used for this purpose include experiments with Rydberg atoms strongly driven by microwave fields [22-28].
Scattering systems with broken time-reversal symmetry were studied thoroughly in experiments with microwave billiards $[29,30]$. The effects of breaking of time-reversal symmetry (TRS) on the spectral properties of the eigenvalues of closed quantum systems have also been investigated in such systems [31-33]. However, it is difficult if not impossible to obtain complete violation of TRS in microwave billiards, whereas its achievement in microwave networks [34-38] is straightforward.

Quantum graphs, introduced by Linus Pauling [39], provide a very useful tool to model many different systems, eg., quantum wires [40], optical waveguides [41] and mesoscopic quantum systems [42, 43]. They consist of vertices connected by $1 \mathrm{D}$ bonds (edges). The BGS conjecture was proven rigourously for graphs with bonds of incommensurable lengths [44, 45]. This was also confirmed experimentally with help of microwave networks [18-20, 34-38].

To perform statistical analysis of the spectral properties of quantum systems on the basis of random-matrix theory (RMT) results for the standard Gaussian ensembles complete sequences of eigenvalues belonging to the same symmetry class are indispensable [5, 46]. Recently, a new procedure to obtain information on the degree of chaoticity of a classical system from the spectral properties of the corresponding quantum system was developed for incomplete sequences of levels [47, 48]. Incomplete spectra pose major problems in real physical systems like, e.g., nuclei and molecules [49-52], which have to be overcome, so such procedures are indispensable for their analysis $[53,54]$. The effect of missing levels is particularly large for long-range spectral fluctuations. R.A. Molina et. al. demonstrated numerically [55] that the power spectrum [56-60] is a powerful statistical measure to discriminate between deviations caused by missing levels and by the mixing of symmetries. Additional information on missing levels may be obtained on the basis of 
commonly used statistical measures for short- and longrange spectral fluctuations [54]. In this paper we present a numerical analysis of missing level statistics for quantum systems with broken time reversal symmetry. For this we use as basis incomplete experimental spectra obtained with fully connected six-vertex microwave network where, additionally, resonance frequencies were removed randomly.

\section{Experimental setup and measurements}

To perform one-port measurements of the scattering matrix $S_{11}$ we used the experimental setup consisting of the Agilent E8364B vector network analyzer (VNA) and a microwave network connected to the VNA via the HP 85133-616 microwave flexible cable, see Fig. 1. Quantum graphs with broken TRS are simulated experimentally by networks of coaxial cables with circulators coupled by junctions at vertices. The coaxial cables (SMA-RG402) consist of an outer concentric conductor of inner radius $r_{2}=0.15 \mathrm{~cm}$, which surrounds dielectric material (Teflon) and an inner conductor of radius $r_{1}=0.05 \mathrm{~cm}$. The dielectric constant of Teflon obtained from the measurements is $\varepsilon \simeq 2.06$. Thus the cut-off frequency of the $T E_{11}$ mode below which only the fundamental $T E M$ can propagate in the cable is $\nu_{c} \simeq \frac{c}{\pi\left(r_{1}+r_{2}\right) \sqrt{\varepsilon}} \simeq 33 \mathrm{GHz}[61,62]$. A circulator is a nonreciprocal passive device. A signal entering the circulator through port 1, 2 or 3 exits at port 2, 3, or 1, respectively. We used the Anritsu PE8403 microwave circulators operating in the frequency range from $7-14 \mathrm{GHz}$. The fully connected, six-vertex networks used in the measurements were composed of fifteen bonds (coaxial cables), four phase shifters, five five-arm joints with circulators, and one six-arm joint connected to the VNA via flexible microwave cable. The geometric lengths of the four bonds was varied with phase shifters (Advanced Technical Materials PNR P1507D) to obtain 30 different realizations of networks. It should be stressed that not the geometric lengths $L_{i}$ but the optical length $L_{i}^{o p t}=L_{i} \sqrt{\varepsilon}$, of the microwave cables yield the lengths of the bonds in the corresponding quantum graph. The total optical length of the networks $L^{o p t}=\sum_{i=1}^{15} L_{i}^{o p t} \simeq 7.2 \mathrm{~m}$ was kept constant.

The scattering matrix $S_{11}$ was measured in the frequency window 7.6-13.0 GHz. The panels (a) and (b) in Fig. 2 show examples of measured reflection spectra in the frequency windows: $7.6-8.6 \mathrm{GHz}$ and $12-13 \mathrm{GHz}$, respectively. According to the Weyl's formula given in [15] $\bar{N}=2 L^{o p t} \nu / c$, where $c$ is the speed of light in the vacuum and $\nu$ is microwave frequency, $\sim 260$ resonances should be observed in the frequency range 7.6-13.0 GHz. On average, in all 30 network realizations, about $3.5 \%$ of resonances were not detected. There are two main reasons for missing resonances in microwave networks: their overlapping with other resonances, which increases with frequency and their small amplitudes. It should be emphasized that the experiments with microwave networks

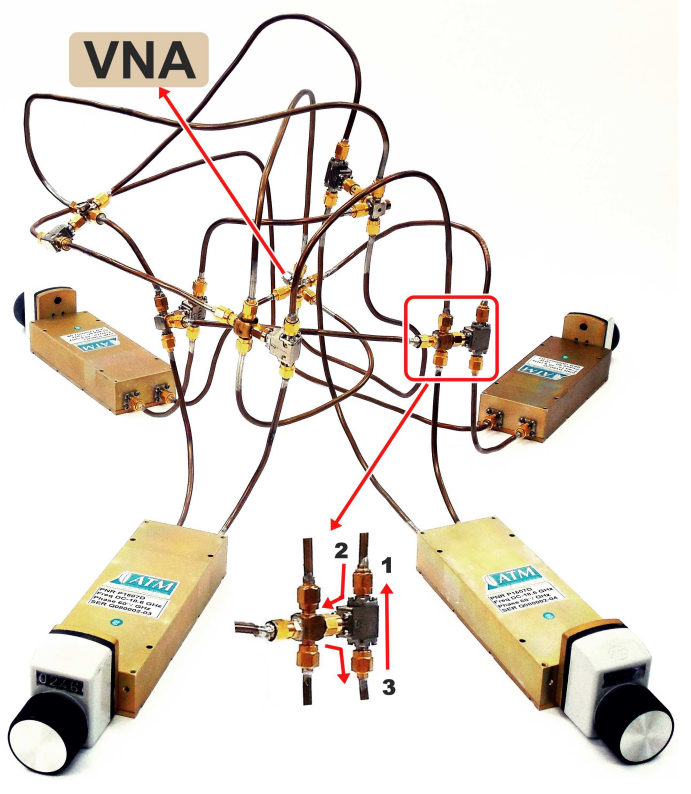

Fig. 1. The six-vertex microwave network containing four phase shifters and five circulators. One vertex with the circulator is shown enlarged at the bottom of the figure.

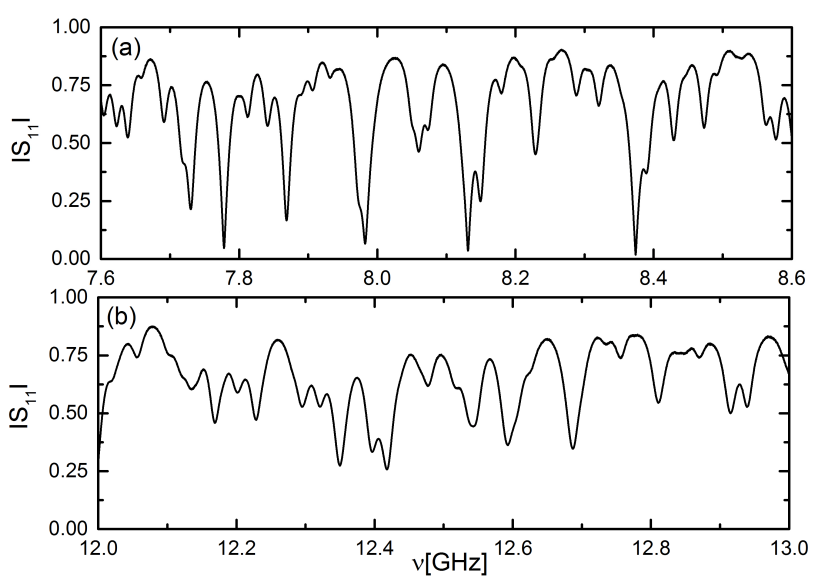

Fig. 2. The examples of the reflection spectra $\left|S_{11}\right|$ in the frequency windows: $7.6-8.6 \mathrm{GHz}$ (panel (a)) and 12$13 \mathrm{GHz}$ (panel (b)), respectively.

provide a unique chance for getting almost complete sequences of resonance frequencies for the purpose of further analysis.

\section{Results}

Before starting with the analysis of spectral properties, the resonance frequencies need to be rescaled (unfolded) to eliminate system specific properties like the total optical length $L^{o p t}$ of the graph. This is done using Weyl's formula. The unfolded eigenvalues determined from the resonance frequencies are given by $\epsilon_{i}=2 L^{o p t} \nu_{i} / c$. 
Before unfolding, we generated three new data series from the spectra obtained with the 30 realizations by removing randomly resonance frequencies from them, yielding sequences with fractions of "missing" resonances equal to $\varsigma=0.075,0.135$, and 0.190 , respectively. The fraction of detected resonances is characterized by the parameter $\phi$, where $0<\phi=1-\varsigma \leq 1$ [54]. The random removal of resonance frequencies was achieved with a Matlab random number generator. These new data were rescaled to mean spacing unity [54] of adjacent resonance frequencies.

The most common measure of the short-range spectral correlations is the nearest-neighbor spacing distribution $P(s)$ describing the distribution of the spacings between adjacent eigenvalues $s_{i}=\epsilon_{i+1}-\epsilon_{i}$ in terms of their mean value $\langle s\rangle$. For long-range spectral correlations we present spectral the rigidity $\Delta_{3}(L)$ which corresponds to the least square deviation of the integrated spectral density of the unfolded $\epsilon_{i}$ from the straight line best fitting it in an interval of length L. We also consider the power spectrum, i.e., the square modulus of the Fourier transform of the deviation $\delta_{q}=\epsilon_{q+1}-\epsilon_{1}-q$ of the spacing between an eigenvalue and its $(q+1)$ st nearest neighbor from its average value $q$.
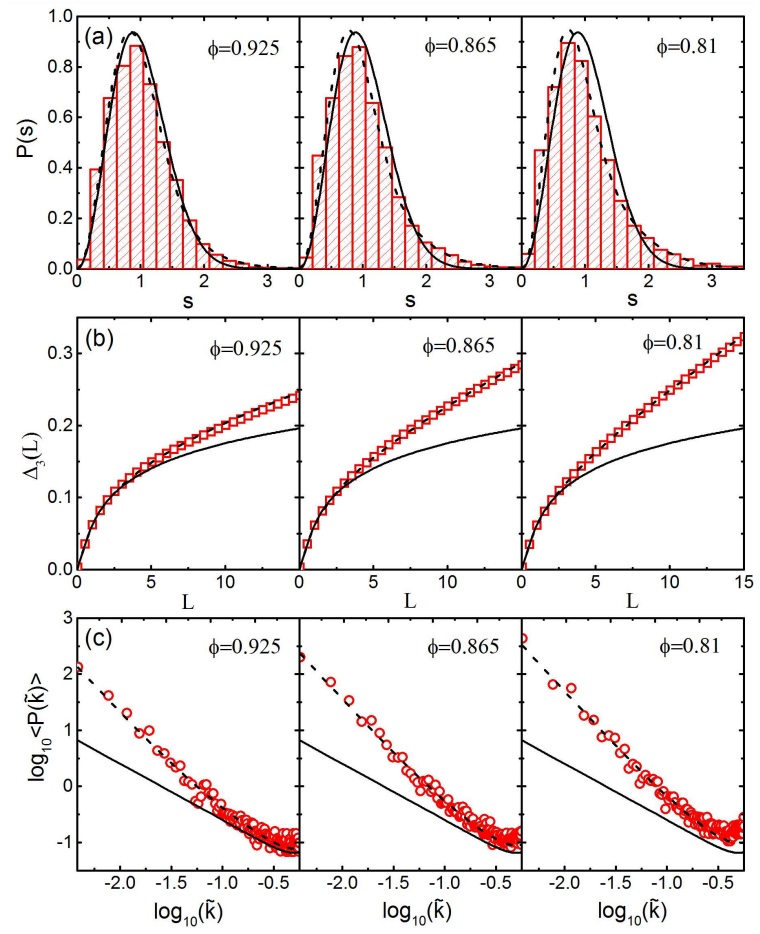

Fig. 3. The nearest-neighbor spacing distribution $P(s)$, the spectral rigidity $\delta_{3}(L)$, and the average power spectrum $P(\tilde{k})$ for three values of $\phi$ are shown in the panels (a), (b), (c), respectively. The modified experimental data are denoted by bars in the panel (a) and by empty circles in the panels (b) and (c) (red empty circles). The theoretical results based on RMT for $\phi=1$, and $\phi<1$ are denoted by solid and broken lines, respectively. The agreement between the modified experimental data and the theoretical ones is good.
The results for the discussed above measures are presented in Fig. 3. The NNSD, the spectral rigidity and the average power spectrum are displayed in the panels (a), (b), and (c), respectively. One should point out that the power spectrum obtained for the incomplete experimental spectra with randomly removed additional resonance frequencies are presented in [47]. The results obtained for the NNSD after removing certain number of resonances from the experimental spectra to achieve $\phi=0.925,0.865,0.810$, respectively, are presented by histograms (red in color) and by empty circles (red in color) in the other panels. To obtain the experimental results for $\phi=0.925,0.865,0.810$ about 7200,6750 , and 6300 eigenvalues from all 30 network realizations were taken into account. The results were obtained by first calculating the statistical measures for each individual network and then averaging over them. The solid and broken lines denote the theoretical results based on random matrix theory (RMT) for complete $\phi=1$, and incomplete series $\phi<1$, respectively.

The NNSD taking into account the incompleteness of a level sequence is given by [54]

$$
P(s)=\sum_{n=0}^{\infty}(1-\phi)^{n} p\left(n, \frac{s}{\phi}\right) .
$$

For complete sequences, $\phi=1$, (solid line in the panel (a)) $P(s)=p(0, s)$, which for GUE systems is well approximated by the Wigner surmise:

$$
P(s)=\frac{32}{\pi^{2}} s^{2} \exp \left(-\frac{4}{\pi} s^{2}\right) .
$$

For $\phi<1$ (broken line in the panel (a)) the following expression was used $P(s) \simeq p\left(\frac{s}{\phi}\right)+(1-\phi) p\left(1, \frac{s}{\phi}\right)+(1-$ $\phi)^{2} p\left(2, \frac{s}{\phi}\right)$, where [32]

$$
p\left(n, \frac{s}{\phi}\right)=\gamma\left(\frac{s}{\phi}\right)^{\mu} \exp \left(-\kappa\left(\frac{s}{\phi}\right)^{2}\right),
$$

with $\mu=7,14$ for $n=1,2$, respectively, and $\gamma$ and $\kappa$ determined from the normalization conditions:

$$
\begin{aligned}
& \int p\left(n, \frac{s}{\phi}\right) \mathrm{d} s=\phi, \\
& \int \frac{s}{\phi} p\left(n, \frac{s}{\phi}\right) \mathrm{d} s=\phi(n+1) .
\end{aligned}
$$

Finally, one gets

$$
\begin{aligned}
& P(s)=\frac{32}{\pi^{2}}\left(\frac{s}{\phi}\right)^{2} \exp \left(-\frac{4}{\pi}\left(\frac{s}{\phi}\right)\right)^{2} \\
& +(1-\phi) \frac{\pi^{4}}{3}\left(\frac{35}{64}\right)^{8}\left(\frac{s}{\phi}\right)^{7} \exp \left(-\pi\left(\frac{35}{64} \frac{s}{\phi}\right)^{2}\right) \\
& \quad+(1-\phi)^{2} \pi^{-8} \frac{6}{7 !}\left(\frac{2^{11}}{9 \cdot 11 \cdot 13}\right)^{16}\left(\frac{s}{\phi}\right)^{14} \\
& \quad \times \exp \left(-\pi^{-1}\left(\frac{2^{11}}{9 \cdot 11 \cdot 13} \frac{s}{\phi}\right)^{2}\right) .
\end{aligned}
$$

The spectral rigidity $\delta_{3}(L)$ in the case of $\phi<1$ (broken 
line in the panel (b)) is given by [54]

$$
\delta_{3}(L)=(1-\phi) \frac{L}{15}+\phi^{2} \Delta_{3}\left(\frac{L}{\phi}\right)
$$

where for $\phi=1$ the spectral rigidity $\Delta_{3}(L)$ (solid line in the panel (b)) is defined by

$$
\begin{aligned}
& \Delta_{3}(L)=\frac{L}{15}-\frac{1}{15 L^{4}} \int_{0}^{L}(L-x)^{3}\left(2 L^{2}-9 x L-3 x^{2}\right) \\
& \times Y_{2}(x) \mathrm{d} x .
\end{aligned}
$$

For GUE systems the two-point cluster function $Y_{2}(x)=$ $\left(\frac{\sin \pi x}{\pi x}\right)^{2}$ [4].

The power spectrum $P(k)=\left|\tilde{\delta}_{k}\right|^{2}$ is the Fourier transform of $\delta_{q}=\epsilon_{i+q}-\epsilon_{i}-q$ from "time" $q$ to $k$ for the sequence of $\mathrm{N}$ levels

$$
\tilde{\delta}_{k}=\frac{1}{\sqrt{N}} \sum_{q=0}^{N-1} \delta_{q} \exp \left(-\frac{2 \pi \mathrm{i} k q}{N}\right) .
$$

It was shown in Refs. $[56,57]$, that for $\tilde{k}=k / N \ll 1$ the average power spectrum exhibits a power law dependence $\langle P(\tilde{k})\rangle \propto(\tilde{k})^{-\alpha}$. Regular systems are characterized by $\alpha=2$ whereas chaotic ones by $\alpha=1$, regardless whether time invariance is preserved or not.

In the case of $\phi \leq 1$ the analytical expression for the average power spectrum [55] is given by

$$
\begin{aligned}
& \langle P(\tilde{k})\rangle=\frac{\phi}{4 \pi^{2}}\left[\frac{K(\phi \tilde{k})-1}{\tilde{k}^{2}}+\frac{K(\phi(1-\tilde{k}))-1}{(1-\tilde{k})^{2}}\right] \\
& +\frac{1}{4 \sin ^{2}(\pi \tilde{k})}-\frac{\phi^{2}}{12}
\end{aligned}
$$

where $0 \leq \tilde{k}=k / N \leq 1$ and $K(\tau)=\tau$ is the spectral form factor for the GUE systems. The avarage power spectrum $\langle P(\tilde{k})\rangle$ for $\phi=0.925,0.865$, and 0.81 , respectively, is shown with broken lines in panel (c) in Fig. 3. The case of $\phi=1$ is plotted in the same panel with the full lines.

The inspection of the results presented in Fig. 3 reveals that for all measures, namely for the nearest-neighbor spacing distribution, the spectral rigidity and the power spectrum, the experimental results are in good agreement with the theoretical predictions based on RMT.

\section{Conclusions}

We compared the nearest-neighbor spacing distribution, the spectral rigidity, and the power spectrum calculated for incomplete experimental spectra, where additionally, resonance frequencies were removed randomly, obtained with six-vertex microwave networks with broken time reversal symmetry to the respective analytical formulas. The agreement between the modified experimental data and the analytical formulas for all these statistical measures are good or very good which clearly shows the power of incomplete spectra analysis.

\section{Acknowledgments}

This work was partially supported by the Ministry of Science and Higher Education grant UMO2016/23/B/ST2/03979 and the EAgLE project (FP7REGPOT-2013-1, Project Number: 316014).

\section{References}

[1] J.M.G. Gómez, K. Kar, V.K.B. Kota, R.A. Molina, A. Relaño, J. Retamosa, Phys. Rep. 499, 103 (2011).

[2] H.A. Weidenmüller, G.E. Mitchell, Rev. Mod. Phys. 81, 539 (2009).

[3] F. Haake, Quantum Signatures of Chaos, SpringerVerlag, Heidelberg 2001.

[4] M.L. Mehta, Random Matrices, Academic Press, London 1990.

[5] O. Bohigas, M.J. Giannoni, C. Schmit, Phys. Rev. Lett. 52, 1 (1984).

[6] M.V. Berry, M. Tabor, Proc. R. Soc. A 356, 375 (1977).

[7] L. Sirko, P.M. Koch, R. Blümel, Phys. Rev. Lett. 78, 2940 (1997).

[8] H.-J. Stöckmann, Quantum Chaos: An Introduction, Cambridge University Press, Cambridge 2000.

[9] Y. Hlushchuk, A. Błędowski, N. Savytskyy, L. Sirko, Physica Scripta 64, 192 (2001).

[10] R. Blümel, P.M. Koch, L. Sirko, Found. Phys. 31 269 (2001).

[11] S. Hemmady, X. Zheng, E. Ott, T.M. Antonsen, S.M. Anlage, Phys. Rev. Lett. 94, 014102 (2005).

[12] B. Dietz, A. Richter, CHAOS 25, 097601 (2015).

[13] M. Ławniczak, M. Białous, V. Yunko, S. Bauch, L. Sirko, Phys. Rev. E 91, 032925 (2015).

[14] B. Dietz, T. Guhr, B. Gutkin, M. Miski-Oglu, A. Richter, Phys. Rev. E 90, 022903 (2014).

[15] T. Kottos, U. Smilansky, Phys. Rev. Lett. 79, 4794 (1997).

[16] T. Kottos, U. Smilansky, Ann. Phys. 274, 76 (1999).

[17] P. Pakoński, K. Życzkowski, M. Kuś J. Phys. A 34 9303 (2001)

[18] O. Hul, S. Bauch, P. Pakoński, N. Savytskyy, K. Życzkowski, L. Sirko, Phys. Rev. E 69, 056205 (2004).

[19] M. Ławniczak, S. Bauch, O. Hul, L. Sirko, Phys. Rev. E 81, 046204 (2010).

[20] M. Ławniczak, S. Bauch, L. Sirko, in Handbook of Applications of Chaos Theory, Eds.: C. Skiadas, C. Skiadas, CRC Press, Boca Raton, USA, 2016, p. 559.

[21] S. Deus, P.M. Koch, L. Sirko, Phys. Rev. E 52, 1146 (1995).

[22] R. Blümel, A. Buchleitner, R. Graham, L. Sirko, U. Smilansky, H. Walther, Phys. Rev. A 44, 4521 (1991).

[23] M. Bellermann, T. Bergemann, A. Haffmann, P.M. Koch, L. Sirko, Phys. Rev. A 46, 5836 (1992).

[24] L. Sirko, S. Yoakum, A. Haffmans, P.M. Koch, Phys. Rev. A 47, R782 (1993).

[25] L. Sirko, P.M. Koch, Appl. Phys. B 60, S195 (1995).

[26] L. Sirko, A. Haffmans, M.R.W. Bellermann, P.M. Koch, Europhys. Lett. 33, 181 (1996).

[27] L. Sirko, S.A. Zelazny, P.M. Koch, Phys. Rev. Lett. 87, 043002 (2001).

[28] L. Sirko, P.M. Koch, Phys. Rev. Lett 89, 274101 (2002). 
[29] B. Dietz, T. Friedrich, H.L. Harney, M. Miski-Oglu A. Richter, F. Schäfer, J. Verbaarschot, H.A. Weidenmüller, Phys. Rev. Lett. 103, 064101 (2009).

[30] B. Dietz, T. Friedrich, H. L. Harney, M. MiskiOglu, A. Richter, F. Schäfer, H. A. Weidenmüller, Phys. Rev. E 81, 036205 (2010).

[31] P. So, S.M. Anlage, E. Ott, R. N. Oerter, Phys. Rev. Lett. 74, 2662 (1995).

[32] U. Stoffregen, J. Stein, H.-J. Stöckmann, M. Kuś, F. Haake, Phys. Rev. Lett. 74, 2666 (1995).

[33] Dong Ho Wu, Jesse S.A. Bridgewater, A. Gokirmak, S.M. Anlage, Phys. Rev. Lett. 81, 2890 (1998).

[34] M. Ławniczak, O. Hul, S. Bauch, P. Seba, L. Sirko, Phys. Rev. E 77, 056210 (2008).

[35] M. Ławniczak, S. Bauch, O. Hul, L. Sirko, Phys. Scr. T143, 014014 (2011).

[36] O. Hul, M. Ławniczak, S. Bauch, A. Sawicki, M. Kuś, L. Sirko, Phys. Rev. Lett. 109, 040402 (2012).

[37] M. Ławniczak, A. Sawicki, S. Bauch, M. Kuś, L. Sirko, Phys. Rev E 89, 032911 (2014).

[38] M. Allgaier, S. Gehler, S. Barkhofen, H.-J. Stöckmann, U. Kuhl, Phys. Rev. E 89, 022925 (2014).

[39] L.J. Pauling, Chem. Phys. 4, 673 (1936).

[40] J.A. Sanchez-Gil, V. Freilikher, I. Yurkevich, A.A. Maradudin, Phys. Rev. Lett. 80, 948 (1998).

[41] R. Mittra, S.W. Lee, Analytical Techniques in the Theory of Guided Waves, Macmillan, NY 1971.

[42] D. Kowal, U. Sivan, O. Entin-Wohlman, Y. Imry, Phys. Rev. B 42, 9009 (1990).

[43] Y. Imry, Introduction to Mesoscopic Physics Oxford, NY 1996.

[44] S. Gnutzmann, A. Altland, Phys. Rev. Lett. 93, 194101 (2004).

[45] Z. Pluhař, H. A. Weidenmüller, Phys. Rev. Lett. 112 144102 (2014)

[46] O. Bohigas, R.U. Haq, A. Pandey, in Nuclear Data for Science, Technology, Ed. K.H. Böckhoff, Reidel, Dordrecht 1983.
[47] M. Białous, V. Yunko, S. Bauch, M. Ławniczak, B. Dietz, L. Sirko, Phys. Rev. Lett. 117, 144101 (2016).

[48] B. Dietz, V. Yunko, M. Białous, S. Bauch, M. Ławniczak, L. Sirko, Phys. Rev. E 95, 052202 (2017).

[49] A. Frisch, M. Mark, K. Aikawa, F. Ferlaino, J. Bohn, C. Makrides, A. Petrov, S. Kotochigova, Nature (London) 507, 475 (2014).

[50] J. Mur-Petit, R.A. Molina, Phys. Rev. E 92, 042906 (2015).

[51] H.I. Liou, H.S. Camarda, F. Rahn, Phys. Rev. C 5, 1002 (1972)

[52] T. Zimmermann, H. Köppel, L.S. Cederbaum, G. Persch, W. Demtröder, Phys. Rev. Lett. 61, 3 (1988).

[53] U. Agvaanluvsan, G.E. Mitchell, J.F. Shriner Jr., M. Pato, Phys. Rev. C 67, 064608 (2003).

[54] O. Bohigas, M. P. Pato, Phys. Lett. B 595, 171 (2004).

[55] R.A. Molina, J. Retamosa, L. Muñoz, A. Relaño, E. Faleiro, Phys. Lett. B 644, 25 (2007).

[56] A. Relaño, J.M.G. Gómez, R. A. Molina, J. Retamosa, E. Faleiro, Phys. Rev. Lett. 89, 244102 (2002).

[57] E. Faleiro, J. M. G. Gómez, R. A. Molina, L. Muñoz, A. Relaño, J. Retamosa, Phys. Rev. Lett. 93, 244101 (2004).

[58] J.M.G. Gómez, A. Relaño, J. Retamosa, E. Faleiro, L. Salasnich, M. Vraničar, M. Robnik, Phys. Rev. Lett. 94, 084101 (2005).

[59] E. Faleiro, U. Kuhl, R.A. Molina, L. Muñoz, A. Relaño, J. Retamosa, Phys. Lett. A 358, 251 (2006).

[60] R. Riser, V. Al. Osipov, E. Kanzieper, Phys. Rev. Lett. 118, 204101 (2017).

[61] D.S. Jones, Theory of Electromagnetism, Pergamon Press, Oxford 1964, p. 254

[62] N. Savytskyy, A. Kohler, S. Bauch, R. Blümel, L. Sirko, Phys. Rev. E 64, 036211 (2001). 